\title{
Multifactorial Causes of Failure to Thrive in a 79-Year-Old Male
}

\author{
Aaron Sacheli ${ }^{a}, b, c$, Veena Panthangi ${ }^{b}$
}

\begin{abstract}
We discuss a case of a 79-year-old Caucasian male with a multifactoral etiology of failure to thrive. Without having any significant past medical problems, the patient's failure to thrive condition initiated following an acute stroke. Over the course of this and future hospitalizations, as well as inpatient care, numerous factors contributing to his failure to thrive were identified. These included: malnutrition, post stroke debility, dysphagia, anemia, depression and malignancy. This report serves to document a complicated case of failure to thrive in a geriatric patient with focused discussion of some etiological contributions to a failure to thrive condition. Additionally, we will describe some salient features pertaining to the clinical management of such cases.
\end{abstract}

Keywords: Palliative care; Geriatric medicine; Failure to thrive; Cachexia; Stroke; Malnutrition; Anemia; Depression; Mirtazapine; Hospice

\section{Introduction}

The etiological basis of geriatric failure to thrive can be classified as organic or psychological, or as in this presented case, both. Malignancy, nonmalignant gastrointestinal (GI) diseases, psychiatric conditions, medication adverse effects, and malnutrition may all contribute to unintentional weight loss in the elderly patient [1]. Those etiological factors, and strategies for effective clinical management as pertinent to this case are the focus of this discussion.

Manuscript accepted for publication August 18, 2014

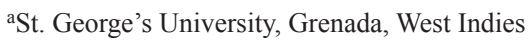

${ }^{b}$ Family Medicine, St. John Hospital \& Medical Center, Detroit, MI, USA

${ }^{c}$ Corresponding Author: Aaron Sacheli, Masonic Medical Center, St. John Hospital \& Medical Center, 21099 Masonic Blvd, St. Clair Shores, MI 48082, USA. Email:asacheli@gmail.com

doi: http://dx.doi.org/10.14740/jmc1923w

\section{Case Report}

The patient is a 79-year-old, Caucasian, widowed male who has been a long-time patient within our primary care facility. Prior to this year, aside from well managed hypothyroidism and hypertension, the patient had no significant past medical problems. Though, significant challenges to his health began to emerge, requiring numerous medical interventions.

Among the first, was a stroke which resulted in left-sided weakness. On presentation to the emergency room, initial laboratory workup revealed a low hemoglobin of $5.8 \mathrm{~g} / \mathrm{dL}$. A low mean corpuscular volume (MCV) of $50.3 \mathrm{fL}$ was found in addition to his anemia with slight poikilocytosis, moderate anisocytosis, and marked hypochromic microcytosis. A further iron panel was obtained which revealed a low iron level $(53 \mathrm{mg} / \mathrm{dL})$ and low iron saturation (19.9\%). The ferritin, total iron binding capacity, and transferrin levels remained within normal limits.

While the possibility of a GI bleed could not be fully ruled out, it was largely hypothesized that the patient's anemia was due to long-standing malnourishment rather than an acute or chronic bleed. Further imaging workup of the stroke etiology, including computed tomography (CT) of the head, CT of the spine and bilateral carotid duplex did not reveal any significant findings.

Considering the likelihood of an existing GI bleed, heparin was not initiated in this patient for ischemic stroke management. He was provided with 3 days of iron (Ferrlecit, 250 mg per day) as well as oral supplementation to be continued out-patient. In addition, the patient was given folic acid and vitamin B12. Throughout the patient's hospital course, the hemoglobin demonstrated an upward trend, reaching $10.0 \mathrm{~g} / \mathrm{dL}$ by discharge.

During the course of this hospitalization the patient received one unit of packed red blood cells on admission and refused a second transfusion. He requested "no resuscitation" at this time. Additionally, for the first time, the patient stated that he did not want treatment or invasive workups. For this reason, palliative medicine as well as hospice care was offered to the patient and his family. While discussed, hospice application was deferred and instead a collective decision was made to first enroll the patient into an extended care facility (ECF) to continue care and post-hospitalization rehabilitation.

Within the ECF, rehabilitation management focused primarily on activities of daily living training, strengthening and 
endurance exercises. It was noted that the patient was improving slightly with rehabilitation. He was able to ambulate with his walker, able to bathe himself and able to change his clothes, for example. However, the patient's continued debility was concerning to the patient's family, and a decision was made to move the patient into his daughter's home so that she could assist in his care.

Six months after this stroke, the patient was seen within our Geriatric Clinic for routine follow-up. Despite scheduled rehabilitation twice weekly, his daughter reported that the patient began to develop new generalized weakness and a difficulty swallowing. A 7.5\% weight loss over 3 months was noted (145.1 lbs to $134.2 \mathrm{lbs}$ ), resulting in a decrease of two body mass index (BMI) points from 24 to $22 \mathrm{~kg} / \mathrm{m}^{2}$ over that time. The daughter stated that the patient had worsening functional debility and was becoming more dependent. In addition, his daughter was chiefly concerned that the patient appeared to have lost his appetite. She attempted to feed the patient two to three bottles of a high calorie nutritional shake per day, with scrabbled eggs as tolerated. While the patient was able to feed himself, the interest in food seemed to be declining. There were also new reports of occasional bowel and urinary incontinence. Routine blood work was gathered including a complete blood count and thyroid function test. A urinalysis was also performed on the suspicion of a urinary tract infection. Additionally, a mental status inventory was obtained on the initial impression of an emerging depressive mood disorder in this patient.

Tests revealed a persistent anemia, with a hemoglobin of $9.8 \mathrm{~g} / \mathrm{dL}$ and an MCV of $75.7 \mathrm{fL}$. Iron supplementation was continued. The thyroid panel showed a TSH which was improved from previous tests $(7.05$ microunits $/ \mathrm{mL})$, and thus levothyroxine $25 \mathrm{mg}$, once daily, was continued. Urinalysis was suggestive of a urinary tract infection as evidenced by trace blood, positive nitrites, and large amount of leukocytes. Resultantly, a course of ciprofloxacin $500 \mathrm{mg}$, twice daily for 7 days was prescribed.

Depressive mood disorder was considered in this patient. This was based on reports from the patient and family of decreased appetite, generally depressed mood on more days than not, the change in living situation post-stroke and subsequent increased reliance on others. We also identified clinically significant weight loss in this patient. This assessment was based on the patient's loss of appetite, reduced caloric intake, weight loss of greater than $7.5 \%$ of body weight over 3 months [2], decreased muscle strength and fatigue. Concern was voiced over a possible oncologic etiology, though the patient's daughter declined further workup at this point. It was now decided that a hospice application should be initiated. Pharmaceutical intervention was started with mirtazapine $15 \mathrm{mg}$ once daily at night-time. The rationale for this pharmacologic strategy was based on the agent's anti-depressant and suggested appetite improving effects.

Two weeks later, the patient presented to our hospital facility following further difficulty swallowing and progressive generalized weakness. Since seen in the out-patient setting, the patient had decreasing oral intake of food and dietary supplements with reports of coughing when swallowing. The patient's weight was largely stable since being seen in the outpatient clinic.

Physically on presentation, the patient was generally cachectic and worse from previous. He had hollow looking orbital areas, scooping of temples, with prominent clavicle and acromion processes. His thighs and calves had very little muscle tone and bones were prominent. The patient was alert and oriented to person only. He had mild scleral icterus and the liver margin was enlarged, extending $6 \mathrm{~cm}$ below the right costal margin, noted to be nodular in texture. His oral mucosa was dry. The remainder of the physical examination was unremarkable. Vital signs showed hypotension $(101 / 50 \mathrm{~mm} \mathrm{Hg}$ ), but were otherwise within normal limits.

Initial laboratory workup included a complete blood count and comprehensive metabolic panel. The patient had hemoglobin of $8.7 \mathrm{~g} / \mathrm{dL}$, with an MCV of $70.7 \mathrm{fL}$ and moderate anisocytosis, with microcytosis and hypochromia. The liver panel revealed elevated total bilirubin $(3.3 \mathrm{mg} / \mathrm{dL})$, direct bilirubin (2.5 mg/dL), alkaline phosphatase (758 units/L), and aspartate transaminase (171 IUnits/L). His albumin was extremely low at $1.6 \mathrm{~g} / \mathrm{dL}$. The alanine transaminase was within normal limits (41 units/L) and subsequently obtained lipase was unremarkable (41 units/L).

Imaging was obtained, including an ultrasound of the abdomen and a chest X-ray. The ultrasound revealed a heterogeneous appearance of the liver suspicious for multiple masses. The chest X-ray showed nodular densities overlying the left side of the chest. Oncologic consultation was pursued, and it was suggested that a malignant process involving his liver and his lungs was the impetus for his presentation, most likely related to his GI tract on the basis of his iron-deficiency anemia. On further discussion with the patient and his family, all were in agreement with directing a more palliative approach and not exploring any further diagnostic workup. The patient's family expressed concern over not being able to fully care for him in his current state, thus extended care was again considered, and further acceleration of the aforementioned hospice application was attempted.

Nutritional consultation was obtained in order to prepare for discharge planning. It was noted that the patient had an oral intake less than $50 \%$ of normal within the past 4 days, and that physical findings indicated severe muscle and fat losses consistent with less than $75 \%$ of estimated energy requirements for greater than 1 month. A daily energy requirement of up to $1,750 \mathrm{kcal} /$ day was recommended, including up to $73 \mathrm{~g}$ of protein. The patient passed a bedside swallow evaluation, though swallow strategies were recommended including bedside at $90^{\circ}$ during meals and having sips of liquid between small bites of soft and pureed foods.

His management over the course of hospitalization largely focused on his dehydrated state, improving oral intake and securing a nutritional plan for discharge. He was discharged after 6 days of hospitalization and died 3 days later.

\section{Discussion}

In elderly adults, failure to thrive is a term used to illustrate 
components of weight loss, decreased appetite, poor nutrition, dehydration and depressive symptoms, among others [3]. It has been suggested that clinically significant weight loss in the elderly meets the following criteria: greater than $2 \%$ decrease in body weight in 1 month, greater than $5 \%$ decrease in 3 months, or greater than $10 \%$ decrease in 6 months [2]. Our patient met the criteria for both the 1 month and 3 months time frames, losing $4.5 \%$ and $7.5 \%$ of body weight respectively.

Malnutrition, as defined from a joint consensus statement from the Academy of Nutrition and Dietetics and American Society for Parenteral and Enteral Nutrition, is based on having two or more of: insufficient energy intake, weight loss, loss of muscle mass, loss of subcutaneous fat, fluid accumulation which may mask weight loss, and diminished hand grip strength [4]. Given documented weight loss, reduced energy intake for 1 month, and objectively observed loss of subcutaneous fat and muscle mass, our patient demonstrated criteria meeting a malnutrition diagnosis. This placed our patient at an already greater risk of mortality, as even a demonstrated weight loss of $5 \%$ or more has been shown to have an increased risk of mortality [5]. As pertaining to our patient, with a starting weight of 145.1 pounds, his age and gender adjusted hazard ratio for mortality would be 2.15 (95\% CI 1.58 - 2.91) [5]. Additionally, with a weight loss starting from a BMI of 24 , this places our patient at additional risk of mortality, given reports of higher 3-year all cause mortality associated with weight loss in elderly patients with BMI below 30 [6]. When malnutrition may not be so apparent as in this case, numerous screening tools exist to asses nutritional status in the elderly patient. Those with the highest sensitivity and specificity [7] are noted to be the Mini Nutritional Assessment [8] and the Malnutrition Screening Tool [9].

The sequela of post-stroke dysphagia can be a central factor to a failure to thrive condition in an elderly patient, and is certainly relevant to our case. In patients with first-ever acute stroke, $51 \%$ and $64 \%$ of them were found to have dysphagia through diagnosis by clinical and video fluoroscopic methods, respectively [10]. Additionally, $49 \%$ and $22 \%$ of patients were found to have evidence of aspiration by those same methods, respectively [10]. While the dysphagia experienced by our patient, may indeterminately have been caused by his first-stroke, it is important to maintain that dysphagia, from any cause, is a preponderant condition in the geriatric population. Reports have suggested approximately 8 to $10 \%$ of the adult population over 50 years of age encounter dysphagia, with rates of up to $40 \%$ in specific populations such as those adults residing in extended care facilities [11]. Undernutrition has been positively associated with dysphagia, in addition to other factors such as poor appetite, low protein intake, and age [12].

Anemia remains a common finding in patients with reduced nutritional intake, decreased muscle strength and fatigue. The presented case here illustrates the comorbid presence of an iron deficiency anemia and hypoalbuminemia, in the context of elderly failure to thrive. It has been proposed that both anemia $(<12 \mathrm{~g} / \mathrm{dL})$ and hypoalbuminemia $(<3.2 \mathrm{~g} /$ dL) be additional factors in the definition of cachexia on the basis of clinical expert opinion and the limited data on patients with cachexia [13].
As a reversible cause of weight loss in the elderly, depression may serve as an impetus for decreased interest in food and decreased appetite, and thus contribute to a failure to thrive condition. Though not statistically significant, it has been noted that depression may cause weight loss in up to $30 \%$ of older patients when compared to their younger counterparts [14]. Antidepressant therapy, with selective-serotonin reuptake inhibitors (SSRIs), remains the forefront for treatment of depression in the elderly. Mirtazapine has been noted as an effective treatment for depression and may serve as an appetite stimulant, though the weight gain effects relative to other SSRIs are not fully clear $[1,15]$. In this presented case, it was acknowledged that depression may have been contributory to the patient's failure to thrive, and that with quantifiable weight loss and appetite diminishment, appetite stimulation would be beneficial. For these reasons, in addition to avoiding multipharmaceutical intervention, mirtazapine was chosen as the antidepressant agent.

Malignancy remains an important medical cause of weight loss among older adults, and should always be given consideration within a differential diagnosis of weight loss. With this case, there was observed malignancy in the liver, and possibility was given to a primary cancer within the GI tract. This explanation for our patient's weight loss is certainly consistent with the literature, as in the majority of malignant causes of weight loss, GI tract neoplasms compromised the greatest proportion $[16,17]$.

Numerous medications may contribute to nutritional deficiency and failure to thrive in the geriatric patient population, and as such, should be considered inappropriate for use in this patient group. Medications which possess anticholinergic properties, especially, should be avoided given their potential for decreased renal clearance, increased risk for confusion, dry mouth, constipation, and urinary retention [18]. The Beers criteria [19] have been developed to assist clinicians in identifying potentially inappropriate medications, and have been heralded as a robust clinical tool intended to improve the selection of medications by the doctor and patient. As pertaining to this case, during each clinical encounter the patient's pharmacologic roster was reviewed for potentially inappropriate medications.

Certainly, weight loss and malnutrition are central to the etiology of failure to thrive in the elderly patient. While the aforementioned etiological factors may contribute, in part, to weight loss and poor nutrition, special attention should also be given to other factors such as assistance needs with eating mechanics, and chewing or swallowing difficulties. Within our presented case, clearly the later factors were contributory to his weight loss. While mechanically able to feed himself, our patient did rely on others for food shopping and preparation, and presented within increasing difficulties of swallowing. Establishing food security, food preparation assistance, and modifying food textures and flavors to adapt for chewing and swallowing debility are strategies to minimize weight loss [20]. The use of oral and nutritional supplements may also help to improve declining weight. The caregiver of our patient did provide a low-volume and high caloric nutritional shake, and strategies of this kind, with paralleled dietary advice, have 
been shown to improve weight, grip strength and muscle circumference, over dietary advice alone [21].

Pharmacologic approaches are also available to assist in stimulating appetite in the geriatric population. Medications such as megestrol acetate and dronabinol are routinely used, though there exists limited evidence on the use of these medication within patients presenting with failure to thrive. Using mirtazapine as a pharmacological choice in elderly patients with a failure to thrive syndrome has captured great interest. Some reports have demonstrated a greater weight gain effect, over other antidepressant agents, in elderly patients with established diagnosis of depression or of dementia with depression [22]. As previously mentioned, weight gain results using mirtazapine have not been consistently demonstrated in studies comprised of a geriatric population. However, the use of mirtazapine as a pharmacologic strategy in geriatric patients with underlying depression, such as in our case, has gained popularity [22]. Namely, due to the effective and well-tolerated profile of mirtazapine as a first-line choice for depression, with a potential weight-gain benefit. In the case presented here, given the multifactorial nature of our patient's failure to thrive condition in addition to the relatively short course of mirtazapine therapy, the appreciation of any weight gain was difficult to elucidate.

While attention to the elderly patient themselves remains central in the medical management of a failure to thrive condition, care should also encompass the needs and concerns of the patient's caregiver. According to relatives of palliative patients, it has been noted that oral intake towards the end of life holds a variety of meaningful traits ranging from nutritional value to social animation [23]. Equally variable, are caregiver's responses to the oral feeding of their palliative relative. Some view their relatives declining oral intake as a self-choice to be respected, while others feel the need to combat a declining oral intake as a method to delay death [23]. Thus, the views of the caregiver toward the feeding habits of their palliative elder, remain an important talking point during clinical encounters. As related here, a discussion with our patient's daughter on her feelings of her father's declining oral intake, served as a vital platform to help the family become aware of the possibility of death. Additionally, this motivated and allowed a seamless transition toward initiating an application to hospice.

With the multifactorial etiology of our patient's failure to thrive, in addition to an aggressive underlying malignancy, cases such as this are limited in the extent of therapeutic repair. Shifting to a palliative care focus, at this stage, is appropriate. With regard to hospice planning, the National Associate for Home Care and Hospice (NAHC), recommends that all related diagnoses are listed on a patient's application to Centers for Medicare and Medicaid Services (CMS), in lieu of solely using "debility" and "adult failure to thrive" as the primary diagnosis [24]. This was attempted in our case. In addition, it is inappropriate to use dementia or other mental, behavioral and neurodevelopment disorders as a principle diagnosis [24]. As in this case, initiating care within a palliative or hospice model allows the values of a patient and their family to be best served during the end-of-life stages.

\section{Conclusions and learning points}

This case report illustrates the mutifactorial etiology of failure to thrive in an elderly male.

In the elderly patient, malignancy, nonmalignant GI diseases, psychiatric conditions, medication adverse effects, and malnutrition may all contribute to unintentional weight loss, as part of the failure to thrive condition [1].

Though the treatment of failure to thrive in the geriatric population may include strategies pertaining to food preparation, food security, pharmacologic approaches and acknowledging the caregiver's views on declining oral intake, the optimal medical management of such cases has yet to be defined.

It remains vital to identify the goals and values of an elderly patient with failure to thrive, and those of their family, in order to engage an appropriate clinical model of care.

\section{Acknowledgement}

The authors wish to thank Debbie Cicchini and Karen Rivera for their assistance in the research of this paper.

\section{Disclosures}

The authors have no conflicts of interest, and no financial disclosures to be made.

\section{References}

1. Gaddey HL, Holder K. Unintentional weight loss in older adults. Am Fam Physician. 2014;89(9):718-722.

2. Zawada ET, Jr. Malnutrition in the elderly. Is it simply a matter of not eating enough? Postgrad Med. 1996;100(1):207-208, 211-204, 220-202 passim.

3. Sarkisian CA, Lachs MS. "Failure to thrive" in older adults. Ann Intern Med. 1996;124(12):1072-1078.

4. White JV, Guenter P, Jensen G, Malone A, Schofield M. Consensus statement: Academy of Nutrition and Dietetics and American Society for Parenteral and Enteral Nutrition: characteristics recommended for the identification and documentation of adult malnutrition (undernutrition). JPEN J Parenter Enteral Nutr. 2012;36(3):275-283.

5. Newman AB, Yanez D, Harris T, Duxbury A, Enright PL, Fried LP. Weight change in old age and its association with mortality. J Am Geriatr Soc. 2001;49(10):13091318.

6. Locher JL, Roth DL, Ritchie CS, Cox K, Sawyer P, Bodner EV, Allman RM. Body mass index, weight loss, and mortality in community-dwelling older adults. J Gerontol A Biol Sci Med Sci. 2007;62(12):1389-1392.

7. Skipper A, Ferguson M, Thompson K, Castellanos VH, Porcari J. Nutrition screening tools: an analysis of the evidence. JPEN J Parenter Enteral Nutr. 2012;36(3):292298.

8. MNA Mini nutritional assessment. www.mna-elderly. 
com. 2014 [cited 2014 Jun 25]. pp. 1-1. Available from: http://www.mna-elderly.com/forms/MNA_english.pdf.

9. Ferguson M, Capra S, Bauer J, Banks M. Development of a valid and reliable malnutrition screening tool for adult acute hospital patients. Nutrition. 1999;15(6):458-464.

10. Mann G, Hankey GJ, Cameron D. Swallowing disorders following acute stroke: prevalence and diagnostic accuracy. Cerebrovasc Dis. 2000;10(5):380-386.

11. Achem SR, Devault KR. Dysphagia in aging. J Clin Gastroenterol. 2005;39(5):357-371.

12. Keller HH. Malnutrition in institutionalized elderly: how and why? J Am Geriatr Soc. 1993;41(11):1212-1218.

13. Evans WJ, Morley JE, Argiles J, Bales C, Baracos V, Guttridge D, Jatoi A, et al. Cachexia: a new definition. Clin Nutr. 2008;27(6):793-799.

14. Wilson MM, Vaswani S, Liu D, Morley JE, Miller DK. Prevalence and causes of undernutrition in medical outpatients. Am J Med. 1998;104(1):56-63.

15. Mihara IQ, McCombs JS, Williams BR. The impact of mirtazapine compared with non-TCA antidepressants on weight change in nursing facility residents. Consult Pharm. 2005;20(3):217-223.

16. Rabinovitz M, Pitlik SD, Leifer M, Garty M, Rosenfeld JB. Unintentional weight loss. A retrospective analysis of 154 cases. Arch Intern Med. 1986;146(1):186-187.

17. Lankisch P, Gerzmann M, Gerzmann JF, Lehnick D. Unintentional weight loss: diagnosis and prognosis. The first prospective follow-up study from a secondary referral centre. J Intern Med. 2001;249(1):41-46.

18. Han L, Agostini JV, Allore HG. Cumulative anticholinergic exposure is associated with poor memory and executive function in older men. J Am Geriatr Soc. 2008;56(12):2203-2210.

19. American Geriatrics Society updated Beers Criteria for potentially inappropriate medication use in older adults. J Am Geriatr Soc. 2012;60(4):616-631.

20. Alibhai SM, Greenwood C, Payette H. An approach to the management of unintentional weight loss in elderly people. CMAJ. 2005;172(6):773-780.

21. Baldwin C, Parsons T, Logan S. Dietary advice for illness-related malnutrition in adults. Cochrane Database Syst Rev. 2007;(1):CD002008.

22. Fox CB, Treadway AK, Blaszczyk AT, Sleeper RB. Megestrol acetate and mirtazapine for the treatment of unplanned weight loss in the elderly. Pharmacotherapy. 2009;29(4):383-397.

23. Raijmakers NJ, Clark JB, van Zuylen L, Allan SG, van der Heide A. Bereaved relatives' perspectives of the patient's oral intake towards the end of life: a qualitative study. Palliat Med. 2013;27(7):665-672.

24. Hospice Claims and Use of Debility, Adult Failure to Thrive, and Dementia Diagnoses. http://www.nahc.org. 2013 [cited 2014 Jun 25]. pp. 1-3. Available from: http:// www.nahc.org/NAHCReport/nr130509_1/. 